\title{
Photonic Bandgap Defect Laser
}

\author{
O. Painter, R. K. Lee, A. Yariv, and A. Scherer \\ California Institute of Technology, Electrical Engineering, \\ MS 136-93, Pasadena, CA 91125, USA \\ E-mail: opainter@cco.caltech.edu \\ J. D. O'Brien, I. Kim, and P. D. Dapkus \\ University of Southern California, Department of Electrical Engineering, \\ Los Angeles, CA 90089
}

In this work we form a microcavity laser using two-dimensional photonic crystals embedded in a half wavelength thick waveguide. Modes localized to a single defect in the photonic crystal can be theoretically shown to have mode volumes as small $2(\lambda / 2 n)^{3}[1]$ and near unity spontaneous emission coupling factors[2]. The flexibility in design of the photonic crystal enables one to tailor the device for vertical emission or for coupling into an in-plane waveguide. These type of devices may be useful for high density, low threshold optical sources in compact optical systems[3]. The added versatility in being able to etch the laser cavity may also help develop low threshold laser sources in material systems in which high index contrast epitaxial mirrors do not exist.

In order to localize the light in all directions we use a combination of a microdisk type structure and a 2D photonic crystal. A thin dielectric slab is used for total-internal reflection of the light in the vertical direction, and a hexagonal array of air holes forms the 2D photonic crystal which provides in-plane localization. The optical cavity itself is created by removing a single hole in the photonic crystal, thereby forming an optical mode localized to the defect region[4]. The modes of the defect cavity have been analyzed previously[i] and consist of a pair of doubly degenerate $\mathrm{x}$ and $\mathrm{y}$ dipole modes. The electric field intensity of the $\mathrm{x}$-dipole mode is shown in Figure 1.

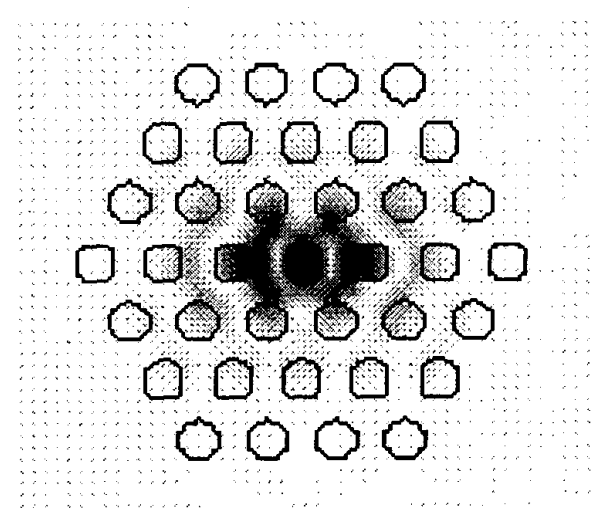

Figure 1: 2D slice through the middle of the slab showing the electric field amplitude of the $\mathrm{x}$-dipole mode. 
Photonic Bandgap..., O. Painter, et. al.

The defect laser cavities were formed in the InGaAsP material system in order to reduce the non-radiative surface recombination rate[5]. The active region consists of four quantum wells (QW) designed for $1.55 \mu \mathrm{m}$ peak emission at room temperature. Electron-beam lithography was used to form the 2D photonic crystal pattern which was then followed by a variety of dry etching techniques to etch the air holes through the active region. Finally, a selective wet etch was used to undercut the active region leaving a free-standing 2D patterned membrane. SEM micrographs of the fabricated defect cavity are shown in Figure 2.

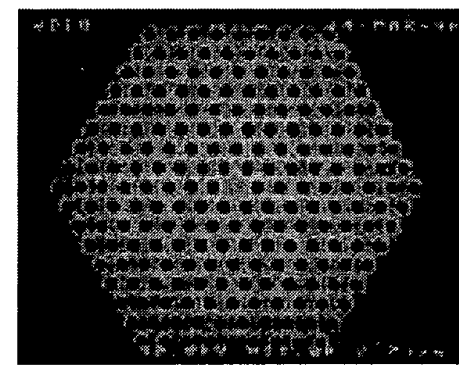

(a)

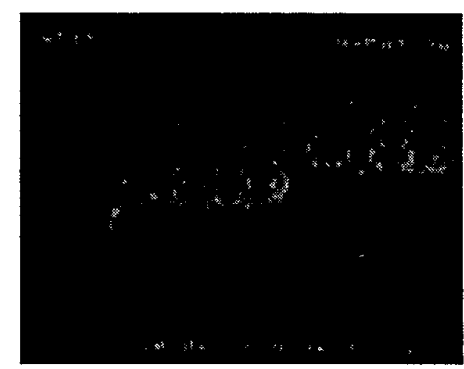

(b)

Figure 2: In (a) we show a top view of a microfabricated 2D hexagonal array of air holes with a single central hole missing. The inter-hole spacing is $500 \mathrm{~nm}$, and the radius of the holes are approximately $150 \mathrm{~nm}$. In (b) a cross-section through the patterned membrane structure is shown.

Lack of thermal heat-sinking in the undercut membranes, along with relatively low modal quality factors, has so far limited lasing action to low temperature, pulsed operation. In this talk we will discuss some of the recent experimental work done on tuning the defect mode frequency lithographically by adjusting the inter-hole spacing of the photonic crystal, as well as the hole radius. By adjusting the defect nearest neighbor holes, one can tune the mode resonance within the (guided mode) bandgap, thus improving the quality factor.

\section{References}

[1] O. Painter, J. Vučković, and A. Scherer, "Defect Modes of a Two-Dimensional Photonic Crystal in an Optically Thin Dielectric Slab," J. Opt. Soc. Am. B 16, 275-285 (1999).

[2] J. Vučković, O. Painter, Y. Xu, A. Yariv, and A. Scherer, finite-difference time-domain calculations of the spontaneous emission coupling factor $(\beta)$ of the defect cavity show that approximately $65 \%$ of the emission is captured by the defect mode when the emission linewidth is as large as $12 \%$ of the peak wavelength. (unpublished).

[3] H. Yokoyama, "Physics and Device Application of Optical Microcavities," Science 256, 66-70 (1992).

[4] P. R. Villeneuve, S. Fan, and J. D. Joannopoulos, "Microcavities in photonic crystals: Mode symmetry, tunability, and coupling efficiency," Phys. Rev. B 54, 7837-7842 (1996).

[5] T. Baba and T. Matsuzaki, "Fabrication and Photoluminescence of GaInAsP/InP 2D Photonic Crystals," Jap. J. Appl. Phys. 1 35, 1348-1352 (1996). 